\title{
Fiberoptic Probe For Hemodynamic Spectroscopy in Freely Moving Mice Shows Cerebrovascular Dysregulation In Alzheimer's and Glioma Models
}

Daniel S. Gareau ${ }^{\mathrm{a} *}$, Matija Snuderl ${ }^{\mathrm{b}}$, Cheddhi Thomas ${ }^{\mathrm{c}}$, N. Sumru Bayin ${ }^{\mathrm{d}}$, Dimitris G. Placantonakis $^{d}$, Julia Zou ${ }^{\mathrm{a}}$, Anna Yaroslavsky ${ }^{\mathrm{e}}$, Michael P. Dietz ${ }^{\mathrm{h}}$, Steven L. Jacques ${ }^{\mathrm{f}}$, Sidney Strickland $^{\text {g }}$, James G. Krueger ${ }^{\mathrm{a}}$, Hyung Jin Ahn ${ }^{\mathrm{h}, \text { *** }}$

(a) Laboratory of Investigative Dermatology, The Rockefeller University, 1230 York Ave, New York, NY 10065, USA

(b) Department of Pathology, NYU Langone Health and Grossman School of Medicine, 550 1st Ave, New York, NY 10016, USA

(c) Department of Neurosurgery, Perlmutter Cancer Center, Kimmel Center for Stem Cell Biology, Neuroscience Institute, NYU Grossman School of Medicine, 550 1st Ave, New York, NY 10016, USA

(d) Developmental Biology Program, Sloan Kettering Institute, 430 East 67th Street, New York, NY 10065, USA

(e) Department of Physics and Applied Physics, University of Massachusetts, Lowell, MA, USA

(f) Bioengineering, University of Washington, Seattle, WA 98195

(g) Laboratory of Neurobiology and Genetics, The Rockefeller University, 1230 York Ave, New York, NY 10065

(h) Department of Pharmacology, Physiology and Neurosciences, Rutgers-New Jersey Medical School, Newark, NJ, 07101, USA

(i) Brain Health Institute, Rutgers University, Piscataway, NJ, 08854, USA

* Daniel Gareau,dgareau@rockefeller.edu

** Hyung Jin Ahn, hyuungin.ahn@ rutgers.edu

\begin{abstract}
:
Significance: Cerebral vascular reactivity is critical parameters of brain homeostasis in health and disease, but the investigational value of brain oxymetry is diminished by anesthesia and mechanical fixation of the mouse scull.
\end{abstract}

Aim: We needed to reduce the physical restrictivity of hemodynamic spectroscopy to enable cancer and Alzheimer's disease (AD) studies in freely-moving mice.

Approach: We combined spectroscopy, spectral analysis software and a magnetic, implantable device to measure vascular reactivity in unanesthetized, freely-moving mice. We measured cerebral blood volume fraction $(\mathrm{CBVF})$ and oxygen saturation $\left(\mathrm{S}_{\mathrm{O} 2}\right)$.

Results: $\mathrm{CBVF}$ and $\mathrm{S}_{\mathrm{O} 2}$ demonstrated delayed cerebrovascular recovery from hypoxia in an orthotopic xenograft glioma model and we found increased CBVF during hypercapnia in a mouse model of AD compared to wild-type littermates.

Conclusions: Our optomechanical approach to reproducibly getting light into and out of the brain enabled us to successfully measure $\mathrm{CBVF}$ and $\mathrm{S}_{\mathrm{O} 2}$ during hypercapnia in unanesthetized freely-moving mice. We present hardware and software enabling oximetric analysis of metabolic activity, which 
provides a safe and reliable method for rapid assessment of vascular reactivity in murine disease models as well as $\mathrm{CBVF}$ and $\mathrm{S}_{\mathrm{O} 2}$.

Keywords: fiberoptic spectroscopy, cerebral oxymetry, Alzheimer's disease mouse model, glioma, cerebrovascular reactivity

\section{Introduction}

\subsection{Importance of cerebrovascular reactivity}

Cerebral perfusion is tightly regulated in response to ischemia (reactive hyperaemia) or the metabolic demand associated with increased local neuronal activation (functional hyperaemia). Vascular reactivity is therefore a fundamental property of blood vessels and enables rapid adjustment of vascular flow to meet the metabolic needs of brain tissue, and is coordinated by the interaction of neurons, glia, endothelium and pericytes(1). Alteration of this response impairs the ability to provide sufficient oxygen and nutrients to the brain and leads to disease states(2). Impaired vascular reactivity has been implicated in Alzheimer's disease $(\mathrm{AD})(2,3)$. AD patients show decreased cerebral hemoglobin oxygen saturation $\left(S_{\mathrm{O} 2}\right)$ during verbal fluency tasks(4) and a failure of cerebral blood flow (CBF) increase during visual stimulus tests(5). Mouse models of $\mathrm{AD}$ also exhibit cerebrovascular dysregulation during whisker stimulation or vasodilator treatment(6). In addition to $\mathrm{AD}$, brain tumors -- particular gliomas -- show high intratumoral vascular heterogeneity with regions of vascular hyperplasia and avascular necrosis interspersed(7). In gliomas, microvascular proliferation in response to tumor hypoxia is closely associated with malignant progression and poor survival(3).

\subsection{Limitations of Current Technology}

Several diagnostic modalities, including functional Magnetic Resonance Imaging (fMRI)(8), functional

Near-Infrared Spectroscopy (fNIRS)(9) and visible light fiberoptic spectroscopy(10), have been used to measure vascular reactivity in animal studies and patients. In animal studies, these techniques require anesthesia in order to minimize movement artifacts, which prevents analysis of cerebral perfusion during behavioral tasks. In addition, anesthesia is known to significantly uncouple neural and vascular responses(11), leading to modified cerebrovascular function. The ability to study changes in cerebral $S_{O 2}$ 
and cerebral blood volume fraction (CBVF) without anesthesia could greatly contribute to a more accurate understanding of oxygen needs and cerebrovascular changes in various neurological disorders.

\subsection{Novel fiberoptic method for measuring $S_{O 2}$ and $C B V F$ in freely moving animals}

In order to overcome these limitations, we developed a device and method for measuring $\mathrm{S}_{\mathrm{O} 2}$ and $\mathrm{CBVF}$ in freely moving animals using fiberoptic spectroscopy. $\mathrm{S}_{\mathrm{O} 2}$ depends on the balance of blood flow and oxygen consumption by the tissue. $S_{O 2}$ and CBVF, which are both unit-less, together characterize the oxygen content in the tissue. Compared to fMRI, fiberoptic spectroscopy is inexpensive and portable, and offers higher temporal resolution. Compared to fNIRS, visible light spectroscopy penetrates only superficially considering the optical scattering of brain tissue (Fig. 1), offering measurement in a banana-shaped volume between a source fiber which illuminates the brain surface and a radiallydisplaced detector fiber (Fig. 2). With deeper implantation of the probe fibers, cerebrovascular measurements of deep brain regions, such as the hypothalamus or amygdala, in response to complex behavioral tasks will be possible with our approach. We developed a connection between the mouse brain and the spectrometer consisting of an implanted brain probe $(\sim 1 \mathrm{~cm}$ fiber $)$ and a spectrometer connector ( $2 \mathrm{~m}$ fiber). The device (Fig. 3a) fiber-optically coupled the brain to the light source and to the spectrometer when magnetically coupled but could be magnetically detached so that the probe could be surgically implanted prior to surgical recovery and subsequent measurement during normal behavior (Fig. 3b,c). After recovery and attachment, spectral measurements were analyzed by least-square fitting to light transport theory (Fig. 3d) to specify the instantaneous $S_{O 2}$ and CBVF. These values varied slightly (Fig. 3e) during normal behavior and CBVF>0 indicated proper attachment reliably.

\section{Materials and Methods}

\subsection{System and computational method of fiberoptic measurement}


A white light source (HL-2000-HP, Ocean Optics, Dunedin FL) illuminated the cerebral cortex through an illumination fiber bundle of 8 fibers (FG105UCA, Thorlabs, Newton, NJ) in 7-around-1 mated to a solid core fiber in the tip (FT400UMT, Thorlabs, Newton, NJ), while a identical detection fiber tip and bundle directed the measured diffuse reflectance to a spectrometer (HDX, Ocean Optics, Dunedin FL). A less sensitive spectrometer (e.g. HR-2000) was used for some experiments but the integration time is much slower $(\sim 0.3 \mathrm{~s})$ so we prefer the high temporal resolution $(\sim 10 \mathrm{~Hz})$ of the HDX instead. Our system was controlled through Matlab (Mathworks, Natick, Massachusetts) software by a laptop computer running Windows 10 . Spectra were fit for real-time readout of $C B V F$ and $S_{O 2}$ on the perioperative laptop during experimentation. The graphic user interface is provided in the supplementary materials.

\subsection{Spectral Calibration}

Spectra were calibrated using measurements of a 99\% reflectance standard (AS-011XX-X60, Labsphere, North Sutton, NH) while holding the fiber probe $3 \mathrm{~cm}$ from the standard. The dark noise of the spectrometer was also measured (dark). Calibrated spectral measurements $M(\lambda)$ were calculated as a function of wavelength $(\lambda)$ from the raw tissue measurements $\mathrm{R}(\lambda)$ and the calibration measurements $\mathrm{C}(\lambda)$ according to Equation 1.

$$
M(\lambda)=\frac{R(\lambda)}{C(\lambda)} \quad M(\lambda)=\frac{R(\lambda)-\operatorname{dark}}{C(\lambda)-\operatorname{dar} k}
$$

where $\mathrm{K}$ is a scaling factor, essentially the ratio of collection efficiency of embedded fibers to collection efficiency of fiber probe held above standard. The measured optical spectrum was dependent on the spectra of the light source and the detector sensitivity but these factors were cancelled by the normalization (Equation 1). A predicted spectrum $\left(M_{p}\right)$ was created by multiplying a predicted $R_{p}$ based on diffusion theory by a scaling factor $(K), M_{p}=K R_{p}$. Equations 2-12 specify the calculation of $R_{p}$, provided here to facilitate reproducibility of $\mathrm{M}_{\mathrm{p}}$ and implement a method we previously developed (10, 
12), which uses the diffusion theory expression of Farrell, Patterson, and Wilson (13-16). The use of this analytical approach (Equations 2-12) was valid, since the $2.5 \mathrm{~mm}$ source-detector separation was large compared to the transport mean free path $\left[1 /\left(\mu_{a}+\mu_{s}^{\prime}\right)=1.26 \mathrm{~mm}\right.$ at $\left.630 \mathrm{~nm}\right]$, and the scattering dominated over absorption.

$$
\begin{aligned}
& r_{i}=0.6681+0.0636 \times n+0.7099 / n-1.4399 / n^{2} \\
& A=\frac{\left(1+r_{i}\right)}{\left(1-r_{i}\right)} \\
& z_{0}=\frac{1}{\mu_{a}(\lambda)+\mu_{s}^{\prime}(\lambda)} \\
& D=\frac{z_{0}}{3} \\
& \Delta=\sqrt{\frac{D}{\mu_{a}(\lambda)}} \\
& r_{1}=\sqrt{z_{0}^{2}+r^{2}} \\
& r_{2}=\sqrt{\left(z_{0}+4 A D\right)^{2}+r^{2}} \\
& \mu_{e f f}=\frac{1}{\Delta} \\
& c=z_{0}\left(\mu_{e f f}+\frac{1}{r_{1}}\right) \times \frac{e^{\frac{-r_{i}}{\Delta}}}{r_{1}^{2}} \\
& d=\left(z_{0}+4 A D\right) \times\left(\mu_{e f f}+\frac{1}{r_{2}}\right) \times \frac{e^{\frac{-r_{2}}{\Delta}}}{r_{2}^{2}} \\
& M_{p}=\frac{c+d}{4 \pi}
\end{aligned}
$$

The fiber separation (distance between the source fiber tip and the detector fiber tip) was $r \approx 0.25 \mathrm{~cm}$, and the refractive index mismatch between glass $\left(\mathrm{n}_{\text {glass }}=1.52\right)$ and tissue $\left(\mathrm{n}_{\text {tissue }}=1.35\right)$ was $n=$ $\mathrm{n}_{\text {glass }} / \mathrm{n}_{\text {tissue }}=1.13 . \mu_{a}(\lambda)$ and $\mu_{s}{ }^{\prime}(\lambda)$ were the absorption and reduced scattering coefficients of the tissue, respectively. The spectral absorption coefficient, $\mu_{a}(\lambda)$, was calculated as a weighted sum of the absorption spectra of oxygenated whole blood $\left(\mu_{a_{-} o x y}(\lambda)\right)$, deoxygenated whole blood $\left(\mu_{a_{-} \text {deoxy }}(\boldsymbol{\lambda})\right)$, and water $\left(\mu_{a_{-} \text {water }}(\lambda)\right)$, according to Equation 13:

$$
\mu_{a}(\lambda)=C B V F \times\left[S_{O 2} \times \mu_{a_{-} o x y}(\lambda)+\left(1-S_{O 2}\right) \times \mu_{a_{-} \text {deoxy }}(\lambda)\right]+W \times \mu_{\left.a_{-} \text {wate } r 13\right)}
$$

\subsection{Calculation of Fitting Parameters to Quantify $S_{O 2}$ and $C B V F$}


Spectra were analyzed in the range 540 to $650 \mathrm{~nm}$ by least-squares fitting. The difference between $M$ (Eq. 1) and $M_{p}=\mathrm{KR}_{\mathrm{p}}$ (see Eq. 12) was minimized by adjusting $M_{p}$ using a multidimensional unconstrained nonlinear minimization method (Nelder-Mead), the fminsearch() in Matlab. Fitting adjusted 3 variables: $C B V F, S_{O 2}$ and $K$ where $C B V F=1$ specifies $150 \mathrm{~g} / \mathrm{L}$ hemoglobin. $W$, the fractional tissue water content, was fixed at an assumed value of 0.78 , which has been reported for mouse cortex (17). Absorption due to water was minimal. For example, at $650 \mathrm{~nm}$, low $\mathrm{CBVF}=0.01$ and $S_{O 2}=0.5$, the absorption coefficient in the tissue due to the presence of blood is $\mu_{a \text {.blood }}=C B V F \times S_{O 2} \times \mu_{a_{-} \text {oxy }}+$ $C B V F \times\left(1-S_{O 2}\right) \times \mu_{a_{-} \text {deoxy }}=0.1103 \mathrm{~cm}^{-1} . \mu_{a_{-} \text {water }}=0.0025 \mathrm{~cm}^{-1}$, which is 44 -fold lower than $\mu_{a . b l o o d}$.

The absorption of the tissue due to blood was specified by the fitting described above while the scattering optical properties (scattering coefficient $\mu_{s}(\lambda)\left[\mathrm{cm}^{-1}\right]$ and scattering anisotropy $g(\lambda)[-]$ ), which were constant across all experiments, were spectral functions $(\lambda)$ fit to the previous measurements of fresh human brain tissue (18). Thus $\mu_{s}(\lambda)$ and $g(\lambda)$ were used as interpolated functions of wavelength (Equations 14-19) that, in combination yielded the reduced scattering coefficient, $\mu_{s}{ }^{\prime}(\lambda)=\mu_{s}(\lambda) \times(1-$ $\mathrm{g}(\lambda)$ ) used in Equation 4. These optical properties agree reasonably with the literature $(18,19)$, such as our previous data (18) that can be fit for the reduced scattering coefficients of gray and white matter as shown in Fig. 1C.

\subsection{Brain optical scattering properties}

Fig. 1 shows the brain scattering properties specified by the spectral data at 5 wavelengths from Yaroslavsky et al. 2002 (18). The Fig. 1a and Fig. 1b show the spectral behavior of the scattering coefficient $\left(\mu_{s}\right)$ and the anisotropy of scattering $(\mathrm{g})$. The combination of $\mu_{s}$ and $\mathrm{g}$ yields the reduced scattering coefficient $\left(\mu_{s}{ }^{\prime}=\mu_{s}(1-\mathrm{g})\right)$ shown in Fig. 1c for white and gray matter. Scattering properties of whole tissue were set as a weighted-sum based on a 1:2 ratio of white to grey matter, as reported (20) for mouse cortex.

$$
\begin{aligned}
& \text { Tissue }{ }_{-} g=\text { WhiteMatter } \_ \text {} g * \frac{1}{3}+\text { GrayMatter }_{-} g * \frac{2}{3} \\
& \text { Tissue } \mu_{s}=\text { WhiteMatter } \mu_{s} * \frac{1}{3}+\text { GrayMatter } \mu_{s} * \frac{2}{3}
\end{aligned}
$$


The $\mu_{s}^{\prime}(\lambda)$ spectrum was assumed when using least-square fitting of the data to specify K, CBVF and $\mathrm{S}_{\mathrm{O} 2}$. After spectral fitting, the values for $C B V F$ and $S_{O 2}$ were output as a function of time (e.g. Fig. 1e) over time periods where spectroscopic measurements were made on mice under various experimental conditions. These optical properties were also used to model the photon transport in the mouse brain.

\subsection{Fiberoptic probe with surgically implantable, magnetically-coupling tip}

The connection between the mouse brain and the spectrometer consisted of an implanted brain probe ( $\sim 1 \mathrm{~cm}$ fiber) and a spectrometer connector ( 2 $\mathrm{m}$ fiber). The connector (Fig. 3a) fiber-optically coupled to the illumination source and the spectrometer and opto-magnetically coupled to the brain probe, which fiber-optically coupled the brain.

Plastic connectors were 3D-printed using a 3D Systems Projet printer and Visijet M3 Crystal material. Mating surfaces were 3D-printed facing upward (opposite the print bed) to minimize warp and maximize surface flatness. The plastic connector design was optimized to align and hold (press fit) two ceramic ferrule cannulas (CF440-10, Thor Labs, Newton NJ) and two cylinder magnets (D12-N52, K\&J Magnetics, Plumsteadville PA). During assembly, the connector's flat surface was pressed against glass, and the ferrules and magnets were inserted and also pressed against the glass for alignment (friction fit). The male side connector was fabricated using a similar process of press fitting the cannulae and magnets, but the assembly was pressed against an alignment tool (see Supplemental Materials), instead of the flat glass plate. The connector was then mated to its probe with magnetic attachment, and prepolished fibers (FT400UMT, Thor Labs, Newton NJ) were inserted into the ferrules and affixed using UV-cure adhesive (\#68, Norland Products, Cranbury NJ). After dry, the protruding fibers were clipped 
and polished to a length of $0.5 \mathrm{~mm}$ protruding from the cannulas using a custom polishing tool set (see Supplemental Fig. 1) that held the probe such that the fibers were normally incident on standard fiber polishing film.

In order to maintain alignment, so that the fibers in the brain probe and spectrometer connector mated precisely, the brain probe surface was printed with 6 small 4-sided pyramids while the connector surface was printed with complementary mating holes. This design configuration enabled secure mating of the two connecting parts during measurements.

Finished fiber probes were visually inspected for polish level and analyzed through a series of signal and noise tests using the spectrometer and the light source targeted on human skin. Human skin was chosen as a biological tissue calibration target because it was readily accessible to the researchers. A threshold for optical performance in satisfactorily-manufactured fiber probes was set as a 60-1 signal-tonoise ratio. The signal was the spectrally summed diffuse remittance with the fiber probe touching skin. The noise, which consisted of light "leaking" directly from the emission fiber of the connector and entering the detection fiber of the connector (ie. not passing through the brain probe), was the sum of the spectrally summed signal measured while pointing the probe into the air in a dark room.

\subsection{Analysis of cerebrovascular reactivity of freely moving mice}

30 mice, over two years during technical development of our probe, were retrofitted with implantable probe tips and measured during normal behavior. 7 month-old C57BL/6 mice (Jackson Laboratory, Bar Harbor, ME) were used to measure basal cerebrovascular dynamic properties of the cortex while freely moving. The AD model mice studied were 13 month-old Tg6799 (Jackson Laboratory) double transgenic mice overexpressing both human amyloid precursor protein (APP) gene with KM670/671/NL, V717I, and I716V mutations and human presenilin 1 (PSEN1) harboring M146L and L286V mutations under the Thy1 promoter (21). Wildtype (WT) littermates were used as controls. Only 
male mice were used in experiments. All mice were maintained in The Rockefeller University's Comparative Biosciences Center and treated in accordance with protocols approved by Rockefeller University's Institutional Animal Care and Use Committee (IACUC). After anesthesia with intraperitoneal injection of ketamine/xylazine $(100 \mathrm{mg} / \mathrm{kg}$ and $10 \mathrm{mg} / \mathrm{kg}$, respectively), mice were mounted on a stereotactic frame. After the removal of hair over the scalp, the scalp was cleaned with sterile alcohol and Betadine three times. We performed a minimal sized midline scalp incision $(\sim 5 \mathrm{~mm})$. We then drilled two small holes in the skull using a $0.7 \mathrm{~mm}$ diameter stainless steel micro drill burr (Fine Science Tools). The coordinates of the two holes were Bregma $=-1 \mathrm{~mm}$, lateral $=1.5 \square \mathrm{mm}$ and Bregma $=-3.5 \mathrm{~mm}$, lateral $=1.5 \square \mathrm{mm}$. Brain probes were fitted to the holes and immobilized using dental cement. Brain probes were fitted to each hole and immobilized using dental cement. The skin incision was sutured and animals were monitored throughout the recovery period. A week after surgery, mice were placed into a chamber $(22.8 \mathrm{~cm} \times 20.3 \mathrm{~cm} \times 15 \mathrm{~cm})$ and we measured cerebral perfusion in the freely moving state. For the hypercapnia study, we injected $10 \% \mathrm{CO}_{2} / 10 \% \mathrm{O}_{2} / 80 \% \mathrm{~N}_{2}$ mixture in to the induction chamber and measured changes in $C B V F$ and $S_{O 2}$ during and after hypercapnia.

\subsection{Analysis of cerebrovascular reactivity in mouse model of glioma}

A separate group of mice were used for the glioblastoma model at the New York University. These mice were housed within NYU School of Medicine's Animal Facilities. Brain tumor implantations were performed according to protocols approved by NYU's IACUC, as previously described (22). Briefly, 68 week-old NOD.SCID (NOD.CB17-Prkdcscid/J, 001303, Jackson Laboratory, Bar Harbor, ME) male mice were anesthetized with intraperitoneal injection of ketamine/xylazine $(100 \mathrm{mg} / \mathrm{kg}$ and $10 \mathrm{mg} / \mathrm{kg}$, respectively). They were then mounted on a stereotactic frame (Harvard Apparatus). A midline skin incision was made. A high-speed drill was used to drill a small hole in the calvaria $2 \mathrm{~mm}$ off the midline and $2 \mathrm{~mm}$ anterior to coronal suture. Five $\mu \mathrm{l}$ of a suspension of U87 cells $(100,000$ cells/ $\mu \mathrm{l})$ were injected through a Hamilton syringe ( $1 \mu \mathrm{l} / \mathrm{min}$, Harvard apparatus, needle pump) into the left frontal lobe through the drilled hole The injection needle was left in place for an additional 5 min after the injection 
was completed to prevent backflow. The skin incision was sutured and animals were monitored throughout the recovery period. U87-MG cells for production of intracranial xenografts were cultured in Dulbecco's Minimal Essential Media (DMEM, Life Technologies) supplemented with 10\% FBS and non-essential amino acids. Previous studies with U87-MG cells in our laboratory showed large tumor formation 2 weeks after injection (22). Two weeks after the injection of cells, terminally ill animals were prepared for fiberoptic measurement. After anesthesia and fitting into the stereotactic frame as described above, we drilled an additional hole in the contralateral frontal calvaria, using a high-speed drill. The diameter of the 2 holes matched the caliber of the probe. After cleaning the area with ethanol wipes, the probes were fitted to each window and immobilized using dental cement. Animals were removed from the stereotactic frame for fiberoptic measurements. We first measured CBVF and $\mathrm{S}_{\mathrm{O} 2}$ at the resting state for $30 \mathrm{sec}$. To analyze the cerebrovascular response during hypoxia, we blocked the nostrils for $30 \mathrm{sec}$ with a wet cotton swap, and measured CBVF and $\mathrm{S}_{\mathrm{O} 2}$ during hypoxia. After nostril blockage release, we continued $\mathrm{CBVF}$ and $\mathrm{S}_{\mathrm{O} 2}$ recordings during reperfusion. This procedure was repeated once, then animals were euthanized following the approved protocol. The skull was opened and the presence of the glioma in situ under the probe was confirmed by a neuropathologist.

\section{Results}

\subsection{Analysis of cerebrovascular reactivity in freely moving C57BL/6 and AD mice}

Fiber spectroscopic measurement of $\mathrm{CBVF}$ and $\mathrm{S}_{\mathrm{O} 2}$ in mixed arterial and venous blood the cortex of freely moving 7-month old C57BL/6 mice yielded values of $\sim 1.4 \%$ and $\sim 55 \%$ respectively (Fig. $4 \mathrm{a}$ ). To investigate the change in $\mathrm{CBVF}$ and $\mathrm{S}_{\mathrm{O} 2}$ of the cortex during hypercapnia in the mouse cortex, we placed mice in an insulated chamber and increased $\mathrm{CO}_{2}$ concentration up to $10 \%$. When hypercapnia was induced after 70 seconds of baseline measurement by increasing $\mathrm{CO}_{2}$ concentration, we found an increase in $\mathrm{CBVF}$, which is a typical hyperaemic response to increased $\mathrm{CO}_{2}$ through vasodilation. $\mathrm{S}_{\mathrm{O} 2}$ decreased slowly but normalized rapidly after stopping $\mathrm{CO}_{2}$ injection and releasing of $\mathrm{CO}_{2}$ at 280 sec 
(Fig. 4b), while CBVF increased rapidly before returning to a level that was less than the baseline measurement.

To investigate the cerebrovascular properties of an $\mathrm{AD}$ (Tg6799) (21) mouse, we measured the baseline of CBVF and $\mathrm{S}_{\mathrm{O} 2}$ of the cortex of freely moving 13 month-old Tg6799 mice and wild-type (WT) littermates and also analyzed the difference of vascular reactivity between Tg6799 mice and WT littermates during and after hypercapnia using fiber optic spectroscopy (Fig. 4c,d). While there is was no difference in baseline of $\mathrm{CBVF}$ and $\mathrm{S}_{\mathrm{O} 2}$ between the two groups, the increase of $\mathrm{CBV}$ of the cortex of AD mice during hypercapnia was significantly higher than that of WT littermates (Fig. 4e). In addition, although not statistically significant, the time to baseline of CBVF of AD mice was also delayed in comparison to WT littermates (Fig. 4f).

\subsection{Analysis of cerebrovascular reactivity in glioma mouse model}

To demonstrate broad utility we analyzed cerebrovascular response to hypoxia in an orthotopic xenograft mouse model generated with human U87 glioblastoma (GBM) cells. Since patients with brain tumors show impaired cerebral vasoreactivity (23-25) and hyperproliferation of immature blood vessels lacking smooth muscle cells limiting perfusion in malignant glioma (3), an orthotopic xenograft glioma mouse model is a good platform to test our new system. Two weeks after surgical implantation of the fiberoptic probe on the skull of control mice (sham injected) and mice orthotopically injected with human U87 GBM cells, we induced hypoxia for 30 seconds and measured the $S_{O 2}$ and $C B V F$ change directly from brain parenchyma. In sham mice, during hypoxia for $30 \mathrm{sec}$, cerebral $S_{O 2}$ decreased but normalized within 30 seconds after hypoxic release (Fig. 4g). We observed the same pattern of hypoxia and rapid normalization at each cycle of obstruction/release as expected for a normal cerebrovasculature. Comparing the $\mathrm{AD}$ recovery time to that of the glioma model mice to the example mouse recovery time for the glioma model, the glioma mouse had a recovery of more than 30 seconds to recover their normal $S_{O 2}$ (Fig. 4h). This result suggests that the cerebrovasculature of glioma has impaired vasoreactivity and reduced ability to restore perfusion after hypoxia. 


\section{Discussion}

Fiberoptic spectroscopy can assess the $S_{O 2}$ and the $C B V F$ in the brains of freely moving mice when implemented with a magnetically coupling tip. The present study showed that our fiberoptic method detected changes in freely moving mice and measured cerebrovascular impairment in a mouse model of GBM. Fig. 4 shows typical $S_{O 2}$ and $C B V F$ values in GBM and background mouse strains. Though our measurements of freely-moving and glioblastoma mice were separate, and we measured only normal mice moving freely and disease mice anesthetized, we hope to measure freely behaving mice of diseasemouse models. $S_{O 2}$ in cerebral blood of control mice was $45-60 \%$, which is comparable to previous studies in the cortex of anesthetized rodents $(26,27) . C B V F$ of 6-8 week old NOD.SCID mice was $2.0 \%$ (Fig. 4a), and CBVF of 7 month old C57BL/6 mice was $1.4 \%$ (Fig. 4b). This range from $1 \%$ to $2 \%$ in our results is slightly less than the range reported in the literature: $2.1 \%$ reported by Synchrotron Radiation Quantitative Computed Tomography in the parietal cortex (28), 2.03-3.14\% reported by MR in the cortex (29-31), 2.0-2.4\% reported by multiphoton laser scanning microscopy and in capillary-rich cerebral cortex (32). We measured $C B V F$ of 7-month old mice, instead of young rodents (4-8 weeks old), which other studies mainly used (29-32).

This fiberoptic method may improve understanding the mechanisms of impaired vascular reactivity in neurologic disorders and brain tumors. Our vasculature and hemodynamic measurements raise the question as to whether the observed phenomena are consequences of the pathology or more fundamental changes that are required for disease progression. We hope to explore these temporal measurements in future work, potentially combining $C B V F$ and $S_{O 2}$ with time-differential mathematics to access perfusion and oxygen consumption parameters. Understanding the extent of impaired vasoreactivity in brain pathologies may someday enable novel diagnostic and prognostics without need for ex-vivo studies. 
Supplementary Materials: The 3D models to print our device, the commercial component part list and the operating system software to replicate our work are described in the supplemental documentation and provided at the following location for download:
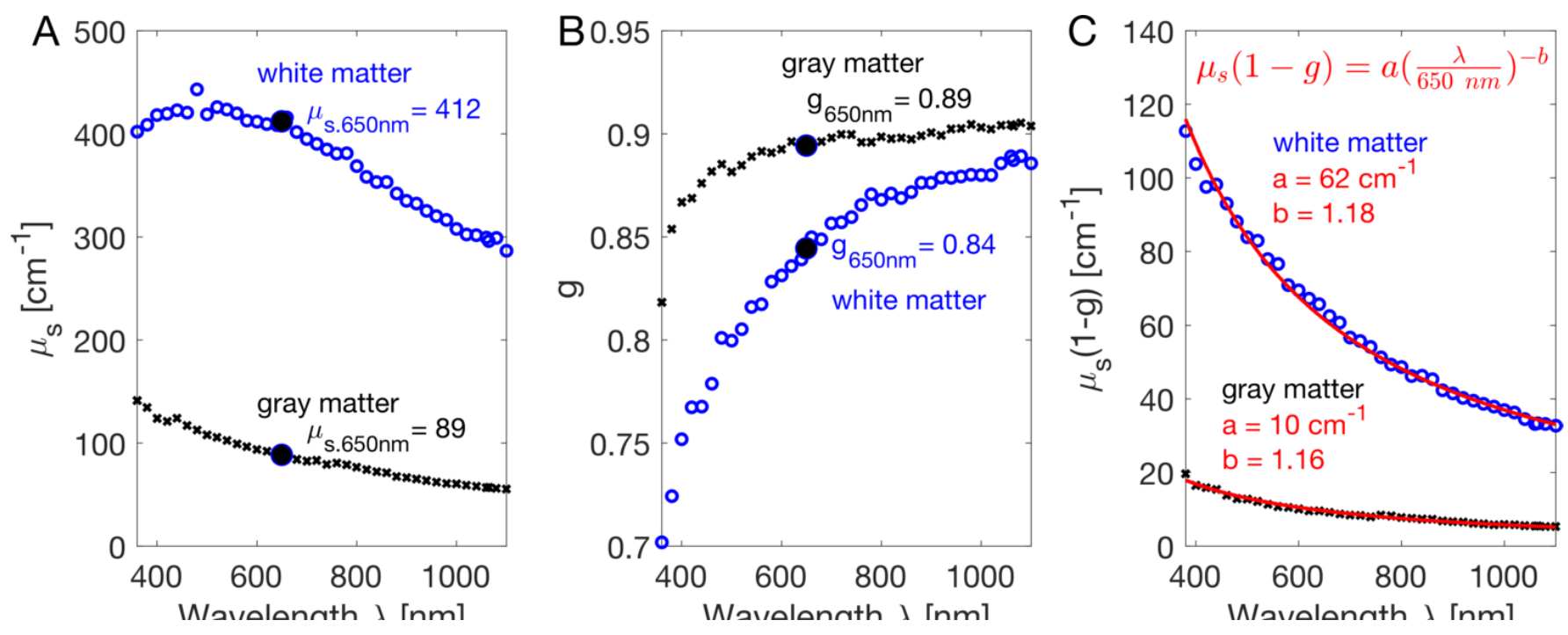

Fig. 1. Spectral scattering coefficient (A) and scattering anisotropy (B) from Yaroslavsky et al.

2002 (18). The fits are combined to yield the spectrum of the reduced scattering coefficient (C), where $\mathrm{a}$ is the $\mu_{s}^{\prime}$ at $650 \mathrm{~nm}$ and $\mathrm{b}$ is the scattering power.

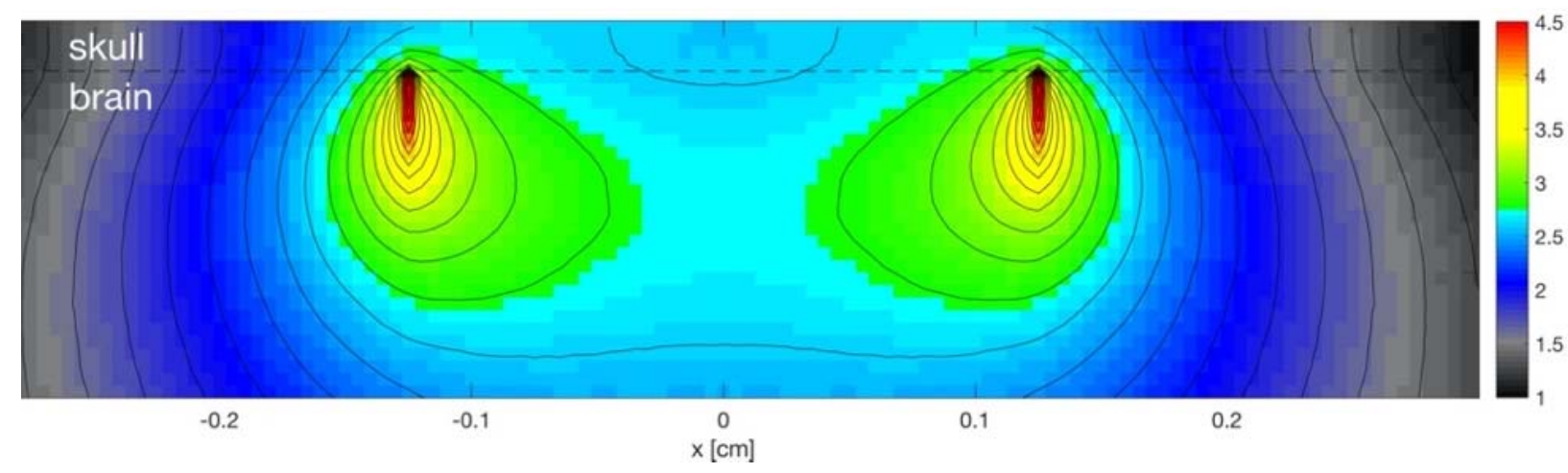

Fig. 2. Monte Carlo simulation outputs a map of the of the surgically implanted probe

The light distribution is shown within the murine skull and brain tissue at $650 \mathrm{~nm}$ wavelength that is both delivered by the source fiber and collected by the collection fiber. The distribution is the joint 
probability of delivering to and collecting from each voxel. $f_{\text {delivered }} \times f_{\text {collected, }}$, which has units of [ $\mathrm{cm}^{-}$ $\left.{ }^{2}\right] \mathrm{x}\left[\mathrm{cm}^{-2}\right]$ or $\left[\mathrm{cm}^{-4}\right]$, where $\mathrm{f}$ denotes fluence $\left[\mathrm{cm}^{-2}\right]$. The figure plots $\log _{10}\left(f_{\text {delivered }} \mathrm{X} \mathrm{f}_{\text {collected }}\right)$.

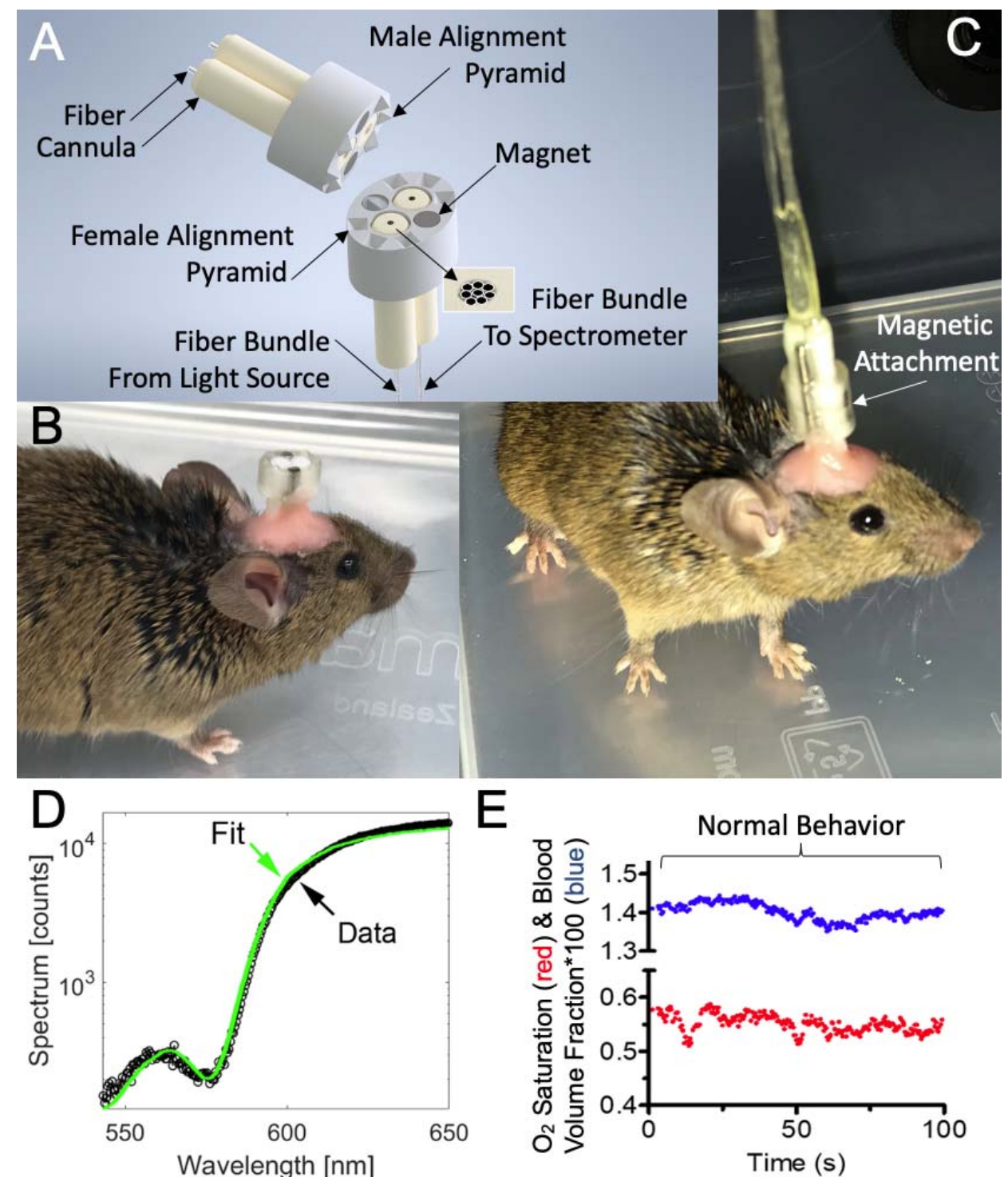

Fig. 3. Instrumentation and typical readout for Fiberoptic cerebral oximetry in freely moving mice.

(A), 3D model of magnetic-coupled probe. 6 male/female mating pyramid pairs secure the detachable, implantable brain probe into a linking fiberoptic with mating female pyramid components to enable fiberoptic alignment. (B), surgically-implanted brain probe. (C), brain probe connected to spectrometer. (D), spectral fitting in $<0.25$ seconds yielding instantaneous measurements of the percentages of CBVF and $\mathrm{S}_{\mathrm{O} 2}$. (E), Typical time-course measurement over normal behavior. The graph shows cerebral blood volume fraction (blue) times 100 such that a y-axis value of 1 means $1 \%$ blood volume fraction and the oxygen saturation fraction (red), which is the fraction of hemoglobin molecules bound to oxygen within the cerebral probed volume. 

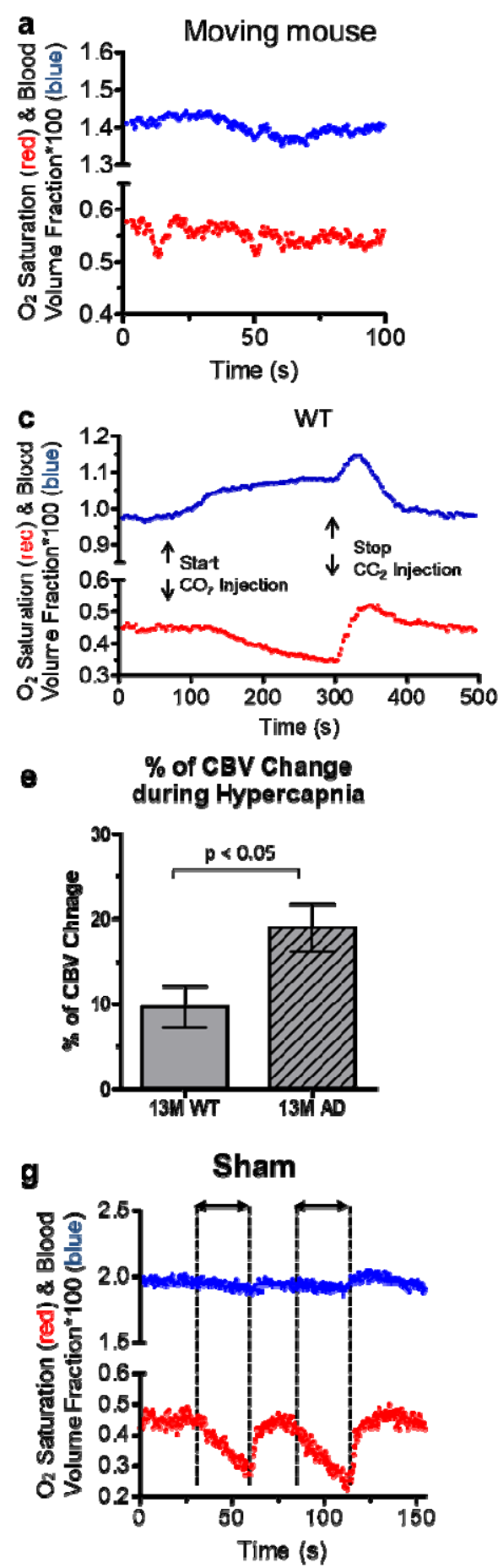
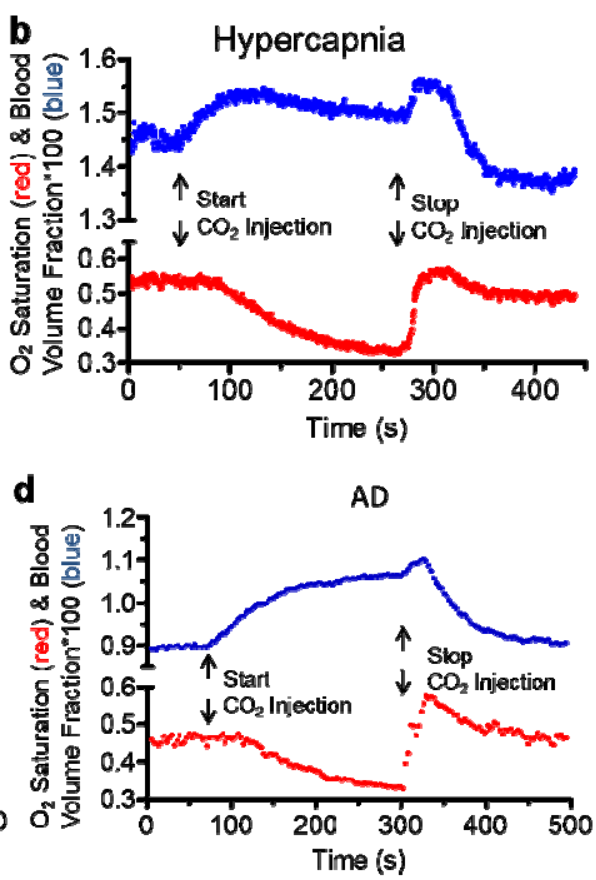

f Time to Basellne of CBV
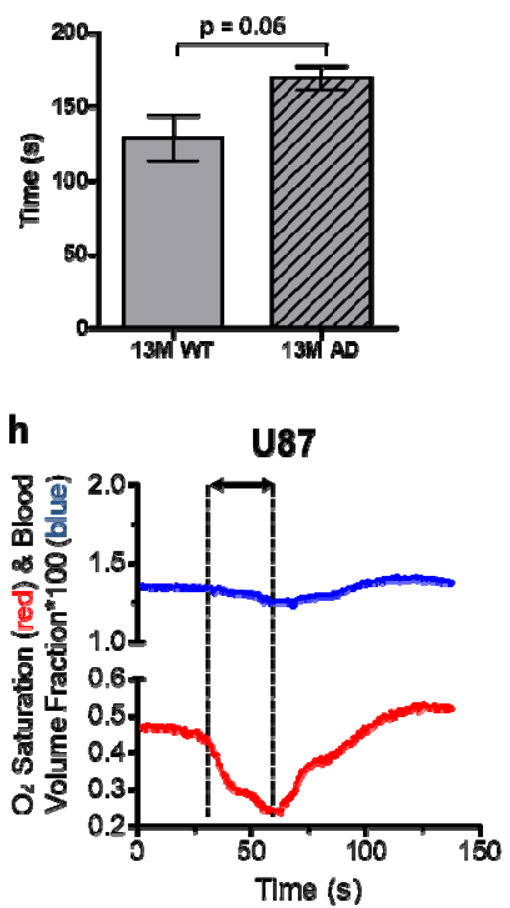

Fig. 4: In vivo spectroscopy results

a, Basal cerebrovascular properties of the cortex of a freely moving, 7 monthold mouse. $b$ Cerebrovascular responses in 7 month-old freely moving mice were obtained using fiberoptic spectroscopy during hypercapnia. Data are representative of three independent experiments. c \& d, Cortical vascular reactivity of $\operatorname{Tg} 679913$ month-old mice (c) and WT littermate (d) during hypercapnia were analyzed using fiberoptic spectroscopy. Hypercapnia was induced at $70 \mathrm{sec}$ by increasing

$\mathrm{CO}_{2}$ concentration up to $10 \%$ and was kept until $300 \mathrm{sec}$. Data are representative of four

independent

experiments. e, The increase of cerebral blood volume fraction (CBVF) during hypercapnia was significantly higher in Tg6799 mice compared to WT littermate $(\mathrm{p}<0.05, \mathrm{n}=4)$. $\mathbf{f}$, The time to baseline of CBV after hypercapnia was also delayed in Tg6799 mice compared to WT littermate, but it is not statistically significant $(\mathrm{p}=0.06, \mathrm{n}=4) . \mathrm{g}$, Hypoxic conditions were held from $30-60 \mathrm{sec}$ and from 80 - $110 \mathrm{sec}$ in 2 month-old mouse. The graph shows cerebral blood volume fraction (blue) and the oxygen saturation fraction (red). $\mathbf{h}$, Delayed Cerebrovascular recovery from hypoxia in a NOD.SCID 2 month- 
bioRxiv preprint doi: https://doi.org/10.1101/2021.05.17.444224; this version posted May 18, 2021. The copyright holder for this preprint (which was not certified by peer review) is the author/funder. All rights reserved. No reuse allowed without permission.

old mouse with intracranial U87 GBM xenograft was delayed compared to a sham mouse. Bar graphs represent mean \pm s.e.m. of $\geq 3$ independent experiments, and was statistically analyzed by performing a two-tailed unpaired t-test. 


\section{ACKNOWLEDGEMENTS}

This work was supported in part by the National Institute of Health NS104386 and UL1 TR000043-09

(Ahn) and in part by the Clinical and Translational Science Award (CTSA) program. Grant Number:

UL1 TR001866. Placantonakis was supported by NINDS R01 NS102665 and NYSTEM IIRP DOH01STEM5-2016-00221/C32595GG.

\section{References:}

1. H. Girouard, and C. Iadecola, "Neurovascular coupling in the normal brain and in hypertension, stroke, and Alzheimer disease," J Appl Physiol (1985) 100(1), 328-335 (2006).

2. C. Iadecola, "Neurovascular regulation in the normal brain and in Alzheimer's disease," Nat Rev Neurosci 5(5), 347-360 (2004).

3. M. E. Hardee, and D. Zagzag, "Mechanisms of glioma-associated neovascularization," Am J Pathol 181(4), 1126-1141 (2012).

4. C. Hock et al., "Decrease in parietal cerebral hemoglobin oxygenation during performance of a verbal fluency task in patients with Alzheimer's disease monitored by means of near-infrared spectroscopy (NIRS)--correlation with simultaneous rCBF-PET measurements," Brain Res 755(2), 293-303 (1997).

5. M. J. Mentis et al., "Visual cortical dysfunction in Alzheimer's disease evaluated with a temporally graded "stress test" during PET," Am J Psychiatry 153(1), 32-40 (1996).

6. L. Park et al., "Nox2-derived radicals contribute to neurovascular and behavioral dysfunction in mice overexpressing the amyloid precursor protein," Proc Natl Acad Sci U S A 105(4), 1347-1352 (2008).

7. Y. Rong et al., "'Pseudopalisading' necrosis in glioblastoma: a familiar morphologic feature that links vascular pathology, hypoxia, and angiogenesis," J Neuropathol Exp Neurol 65(6), 529-539 (2006).

8. A. Niranjan et al., "fMRI mapping of the visual system in the mouse brain with interleaved snapshot GEEPI," Neuroimage 139(337-345 (2016).

9. S. Lee et al., "Cerebral hemodynamic responses to seizure in the mouse brain: simultaneous near-infrared spectroscopy-electroencephalography study," J Biomed Opt 15(3), 037010 (2010).

10. D. S. Gareau et al., "Optical fiber probe spectroscopy for laparoscopic monitoring of tissue oxygenation during esophagectomies," J Biomed Opt 15(6), 061712 (2010).

11. K. Masamoto, and I. Kanno, "Anesthesia and the quantitative evaluation of neurovascular coupling," $J$ Cereb Blood Flow Metab 32(7), 1233-1247 (2012).

12. T. H. Pham et al., "Decreased conduit perfusion measured by spectroscopy is associated with anastomotic complications," Ann Thorac Surg 91(2), 380-385 (2011).

13. T. J. Farrell, M. S. Patterson, and B. Wilson, "A diffusion theory model of spatially resolved, steady-state diffuse reflectance for the noninvasive determination of tissue optical properties in vivo," Med Phys 19(4), 879-888 (1992).

14. R. A. Groenhuis, J. J. Ten Bosch, and H. A. Ferwerda, "Scattering and absorption of turbid materials determined from reflection measurements. 2: measuring method and calibration," Appl Opt 22(16), 24632467 (1983).

15. R. A. Groenhuis, H. A. Ferwerda, and J. J. Ten Bosch, "Scattering and absorption of turbid materials determined from reflection measurements. 1: theory," Appl Opt 22(16), 2456-2462 (1983).

16. W. Egan, Optical Proerties of Inhomogenous Materials, Academic Press (1979).

17. T. Nakamura et al., "Intracerebral hemorrhage in mice: model characterization and application for genetically modified mice," J Cereb Blood Flow Metab 24(5), 487-494 (2004).

18. A. N. Yaroslavsky et al., "Optical properties of selected native and coagulated human brain tissues in vitro in the visible and near infrared spectral range," Phys Med Biol 47(12), 2059-2073 (2002).

19. S. L. Jacques, "Optical properties of biological tissues: a review," Phys Med Biol 58(11), R37-61 (2013).

20. K. Zhang, and T. J. Sejnowski, "A universal scaling law between gray matter and white matter of cerebral cortex," Proc Natl Acad Sci U S A 97(10), 5621-5626 (2000). 
21. H. Oakley et al., "Intraneuronal beta-amyloid aggregates, neurodegeneration, and neuron loss in transgenic mice with five familial Alzheimer's disease mutations: potential factors in amyloid plaque formation," J Neurosci 26(40), 10129-10140 (2006).

22. N. S. Bayin et al., "Selective lentiviral gene delivery to CD133-expressing human glioblastoma stem cells," PLoS One 9(12), e116114 (2014).

23. J. H. Chan et al., "Discrimination of an infected brain tumor from a cerebral abscess by combined MR perfusion and diffusion imaging," Comput Med Imaging Graph 26(1), 19-23 (2002).

24. J. M. Provenzale, S. Mukundan, and D. P. Barboriak, "Diffusion-weighted and perfusion MR imaging for brain tumor characterization and assessment of treatment response," Radiology 239(3), 632-649 (2006).

25. Z. Jiang et al., "Impaired fMRI activation in patients with primary brain tumors," Neuroimage 52(2), 538548 (2010).

26. E. Vovenko, "Distribution of oxygen tension on the surface of arterioles, capillaries and venules of brain cortex and in tissue in normoxia: an experimental study on rats," Pflugers Arch 437(4), 617-623 (1999).

27. S. Sakadzic et al., "Two-photon high-resolution measurement of partial pressure of oxygen in cerebral vasculature and tissue," Nat Methods 7(9), 755-759 (2010).

28. J. F. Adam et al., "Absolute cerebral blood volume and blood flow measurements based on synchrotron radiation quantitative computed tomography," Journal of cerebral blood flow and metabolism : official journal of the International Society of Cerebral Blood Flow and Metabolism 23(4), 499-512 (2003).

29. J. F. Dunn et al., "Monitoring angiogenesis in brain using steady-state quantification of DeltaR2 with MION infusion," Magn Reson Med 51(1), 55-61 (2004).

30. S. Huang et al., "Cerebral blood volume affects blood-brain barrier integrity in an acute transient stroke model," Journal of cerebral blood flow and metabolism : official journal of the International Society of Cerebral Blood Flow and Metabolism 33(6), 898-905 (2013).

31. S. H. Han et al., "Robust MR assessment of cerebral blood volume and mean vessel size using SPIONenhanced ultrashort echo acquisition," Neuroimage 112(382-389 (2015).

32. P. Verant et al., "A direct method for measuring mouse capillary cortical blood volume using multiphoton laser scanning microscopy," Journal of cerebral blood flow and metabolism : official journal of the International Society of Cerebral Blood Flow and Metabolism 27(5), 1072-1081 (2007). 